\title{
Adolescent Preventive Health Visits: A Comparison of Two Invitation Protocols
}

\author{
Barry Knishkowy, MD, MPH, Hava Palti, MD, MPH, Moshe Schein, MBChB, MS, \\ Jobn Yaphe, MD, Richard Edman, MD, and Mario Baras, PbD
}

Background: Adolescent health care in family practice at times creates conflicting responsibilities for parents and their teenagers. In the context of a new adolescent preventive health program in a family practice setting, we compared attendance rates using two invitation protocols, the protocols differing in their emphasis on adolescent autonomy vs parental responsibility.

Metbods: One hundred six teenagers in the seventh and tenth grades were invited for preventive health visits with the family nurse and physician using two protocols. Protocol 1 involved obtaining parental consent before approaching the adolescent. With protocol 2 , an invitation letter and parental consent form were mailed to the teenager, while a letter of explanation was sent concurrently to the parents. In each case, the letter of invitation was followed up by a telephone call for those who did not respond. The spontaneous response rate (a positive response after receiving the letter), agreement to attend rate (a positive response after receiving the letter or being telephoned), and the attendance rate were determined according to grade, sex, and protocol.

Results: The spontaneous response rate was $21 \%$, the agreement to attend rate was $75 \%$, and the attendance rate was $44 \%$. Attendance rates were higher for the girls compared with the boys (54\% vs $35 \%, P=.08)$ and for the seventh graders compared with the tenth graders $(53 \%$ vs $31 \%, P=.03)$. The spontaneous response rate was lower among the tenth graders using protocol 2 (8\% vs $37.5 \%$ with protocol $1, P=.04$ ), while the agreement to attend rate and attendance rate did not differ for the two protocols.

Conclusions: Nearly one half of this population of adolescents attended preventive health visits at the family nurse's and physician's initiative. A follow-up telephone call after the initial written invitation resulted in increased participation, while approaching the teenager or parent initially did not make a difference in attendance. This pilot study shows the potential for initiating an adolescent health program in the family practice setting. (J Am Board Fam Pract 2000;13:11-6.)

Adolescent preventive health visits in primary care have a great potential for screening and providing counseling regarding the major health issues of teenagers. Only recently have comprehensive guidelines for such visits been available to practitioners. ${ }^{1}$ Many questions remain unanswered regarding the content and process of such visits. ${ }^{2}$

For family physicians, some of these questions have unique relevance related to caring for both

Submitted, revised, 4 October 1999.

From the Family Medicine (BK, MS, JY, RE) and Maternal and Child Health (HP) Units; Department of Social Medicine; Hadassah Medical Organization and the Braun School of Public Health and Community Medicine of the Hebrew University and Hadassah, Jerusalem, Israel. Address reprint requests to Barry Knishkowy, MD, MPH, Mother Child and Adolescent Health Department, Ministry of Health, 20 King David Street, P.O.B.1176, Jerusalem 91010 , Israel.

This study was supported by a grant from the Israel Ministry of Health. parents and their teenaged children. In initiating preventive adolescent health visits, for example, issues arise regarding parental consent, confidentiality, and whether parent or child should be contacted for scheduling these visits. These issues have an impact on the success of the interactions and on compliance or attendance.

In Israel, preventive health care visits for adolescents are generally not performed. We have developed and implemented the first adolescent health program in an Israeli family practice (the Tamar Program). In the early stages of the program, we tested two different invitation protocols. These protocols differed in whether parental consent was obtained before approaching the adolescent. They were tested because of the staff's sense of conflicting responsibilities and uncertainty about how parents and teenagers would react to the two approaches. Our hypothesis was that attendance would be enhanced with an initial approach to the 
Table 1. Content of Comprehensive Health Visits Major Health Topics.

Individual concerns growth and development

Dental health

Nutrition and physical activity

Psychosocial adjustment

Smoking, alcohol and substance abuse

Depression

Injury prevention

Sexual behavior (grade 10 only)

teenager. In this article we report on initial compliance data using these two different protocols.

\section{Methods}

The Jerusalem Tamar Program is a communityoriented preventive adolescent health program for patients enrolled in a family practice clinic. ${ }^{3}$ In this program, all teenagers in the practice are invited for comprehensive health visits with their family physician and nurse when they are in the seventh and tenth grades. The visits include a self-administered health questionnaire, a biomedical and psychosocial health assessment, ${ }^{4}$ counseling regarding various adolescent health issues and individual concerns (Table 1), and a brief physical examination. The program and study were approved by the Research Ethics Committee of the Hadassah University Hospital.

The family practice clinic is located in an urban, mainly lower-middle class, Jewish neighborhood in West Jerusalem. All patients in the practice have national health insurance coverage, and all clinic visits are free of charge. The family practice staff includes 4 physician-nurse teams, each of which is responsible for the ongoing care of a defined population. The target population for the program includes approximately 240 teenagers aged 12 to 18 years who live with their parents in the neighborhood. The target population for this study was a cohort of 106 teenagers who were in the seventh and tenth grades during the school years 1994-5 and 1995-6.

The two invitation protocols used were as follows:

Protocol 1 (scbool year 1994-5): The family physician and nurse sent a letter of explanation, consent form, and health questionnaire to the teenager's parents. The parents were requested to return the consent form and questionnaire to the staff, after which the nurse would call the teenager to set $\frac{c}{3}$ up an appointment. After a minimum of 3 weeks, the nurse telephoned parents who had not re- $\frac{0}{2}$ sponded to remind them to return these materials 7 and to ask their permission to invite their child for the health visit. With the parent's consent, the teenager was then invited by telephone.

Protocol 2 (school year 1995-6): A letter of explanation, without the consent form and with a much ${ }_{C}$ shorter questionnaire, was sent to the parents (Ap- $\frac{\bar{\sigma}}{\bar{c}}$. pendix 1). Simultaneously, an invitation letter, in- $\widehat{\mathbb{\Phi}}$ cluding a consent form to be signed by the parents, was sent to the teenager (Appendix 2). Appoint- $\vec{\circ}$ ments were scheduled for those teenagers who responded or whose parents responded for them. $\stackrel{N}{N}$ After a minimum of 3 weeks, the nurse called every teenager who had not responded to set up an appointment.

All seventh grade patients were invited during $\overrightarrow{\vec{\Delta}}$ both years of the study. Tenth grade patients of two 윽 teams were invited during the first year (the other two teams were excluded because of time constraints) and tenth grade patients of all four teams were invited during the second year of the study. $N$

In this preliminary analysis of compliance, the following rates were compared for the two invitation protocols: the spontaneous response rate (the percentage of parents or teenagers who called after receiving the letters); the agreement to attend rate $\frac{}{\Phi}$ (the percentage of teenagers who agreed to attend $\underset{\overrightarrow{\vec{F}}}{\overrightarrow{2}}$ after receiving the letters, or after being called by the family nurse); and the attendance rate (the percentage of teenagers who ultimately came for the health visit). These data are presented by grade and sex.

The Fisher's exact test was used to test for dif- $\frac{}{3}$ ferences in these rates according to protocol, grade $\stackrel{0}{0}$ and sex. ${ }^{5}$

\section{Results}

A total of 106 teenagers were invited for health visits during the first 2 years of the program. Forty- N seven adolescents were invited during the 1994-5 $\mathrm{W}$ school year (protocol 1). There were 31 seventh graders and 16 tenth graders, of whom 29 were $\stackrel{0}{\Phi}$ boys and 18 were girls. In the $1995-6$ school year, $\stackrel{?}{?}$ 59 adolescents were invited (protocol 2). There were 33 seventh graders and 26 tenth graders, of whom 25 were boys and 34 were girls.

The overall spontaneous response rate was $21 \%$ : $20 \%$ for boys and $21 \%$ for girls, and $22 \%$ for 8 
Grade 7, $n=64$
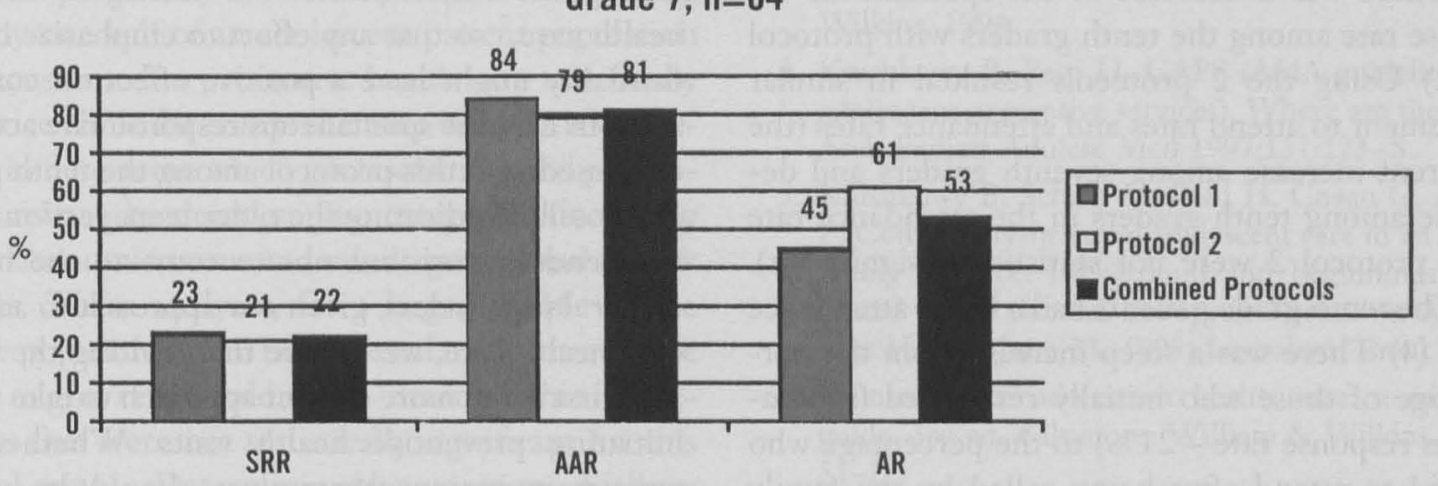

Grade $10, n=42$
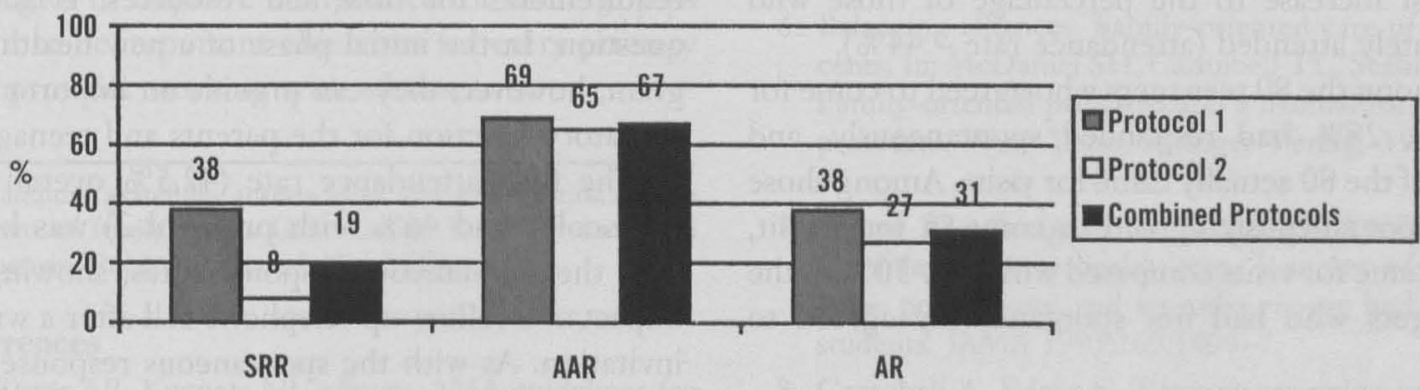

Combined Grades, $n=106$

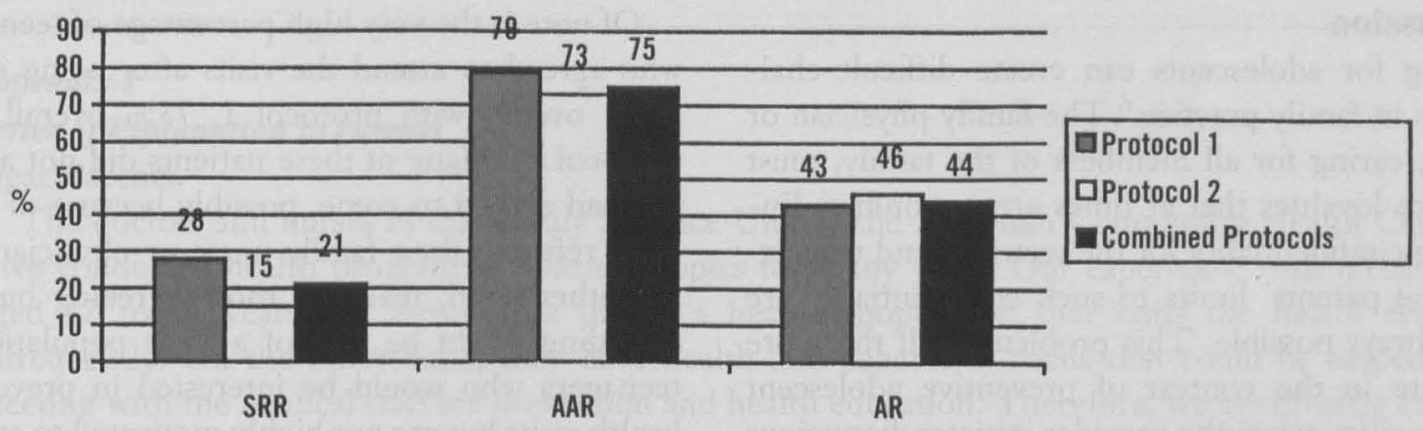

Figure 1. Response to adolescent health unit invitations (percentages) by invitation protocol and grade. Protocol 1 $(n=47)$, invitation and consent form sent to parents; protocol $2(n=59)$, invitation and parental consent form sent to teenager; combined protocols $(n=106)$. SRR - spontaneous response rate, AAR - agreement to attend rate, AR - attendance rate.

seventh graders and $19 \%$ for tenth graders. For the seventh graders the spontaneous response rate was $23 \%$ using protocol 1 and $21 \%$ using protocol 2 . For the tenth graders it was $37.5 \%$ using protocol 1 and $8 \%$ using protocol 2 , the only difference that reached statistical significance $(P=.04)$.

The overall agreement to attend rate was $75 \%$. The rate was $74 \%$ for boys and $77 \%$ for girls, and $81 \%$ for seventh graders and $67 \%$ for tenth graders. For the seventh graders the agreement to attend rate was $84 \%$ using protocol 1 and $79 \%$ using protocol 2. For the tenth graders it was $69 \%$ using protocol 1 and $65 \%$ using protocol 2. None of these differences reached statistical significance.

The overall attendance rate was $44 \%$. The rate was $35 \%$ for boys and $54 \%$ for girls $(P=.08)$, and $53 \%$ for seventh graders and $31 \%$ for tenth graders $(P=.03)$. For the seventh graders the attendance rate was $45 \%$ using protocol 1 and $61 \%$ using protocol 2. For the tenth graders it was $37.5 \%$ using protocol 1 and $27 \%$ using protocol 2 . The last two differences were not statistically significant.

Figure 1, which displays these rates by protocol and by grade, illustrates some of the main findings. 
(1) There was a decrease in the spontaneous response rate among the tenth graders with protocol 2. (2) Using the 2 protocols resulted in similar agreement to attend rates and attendance rates (the apparent increase among seventh graders and decrease among tenth graders in the attendance rate with protocol 2 were not statistically significant). (3) The tenth grade patients had a lower attendance rate. (4) There was a steep increase from the percentage of those who initially responded (spontaneous response rate $-21 \%$ ) to the percentage who agreed to attend after being called by the family nurse (agreement to attend rate $-75 \%$ ), and a more modest increase to the percentage of those who ultimately attended (attendance rate $-44 \%$ ).

Among the 80 teenagers who agreed to come for a visit, $28 \%$ had responded spontaneously, and $59 \%$ of the 80 actually came for visits. Among those who spontaneously agreed to come in for a visit, $82 \%$ came for visits compared with only $50 \%$ of the teenagers who had not spontaneously agreed to visit.

\section{Discussion}

Caring for adolescents can create difficult challenges in family practice. ${ }^{6}$ The family physician or nurse, caring for all members of the family, must balance loyalties that at times are in conflict. Ensuring confidentiality for the teenager and respecting the parents' limits to such confidentiality are not always possible. This problem is all the more delicate in the context of preventive adolescent health visits, when the provider initiates discussions about sexuality, depression, drug abuse, and other sensitive issues.

We have initiated the first program of comprehensive adolescent visits in an Israeli family practice. To involve the parents and to avoid overstepping boundaries, our staff decided to implement an invitation protocol, using a written explanation and questionnaire that would first ask for the parents' input and approval. Only afterward would we invite their children.

Response to our initial invitations, sent by mail (spontaneous response rate), was low (28\% overall). We assumed that using protocol 2, which gave the teenagers responsibility for scheduling their own visits, and thereby fostered adolescent autonomy, there would be increased compliance. It should be recalled that concern regarding confidentiality is among the major barriers to teenagers' seekin health care, ${ }^{7}$ so that any effort to emphasize conffo dentiality might have a positive effect on complö ance. In fact, the spontaneous response rate actually decreased with this protocol among the tenth grad $\beta$ ers, possibly indicating the older teenagers' makin independent decisions not to come to the healtf center. Nonetheless, given our approach to adoles $\vec{\Rightarrow}$ cent health care, we believe that inviting the teer? ager first is the more correct approach to take whe initiating preventive health visits. Whether th written invitations themselves should be elimis nated, given the low response rate and the extro requirements for time and resources, is anotheis question. In the initial phase of a new health pros gram, however, they can provide an important exo planatory function for the parents and teenagers. $\overrightarrow{3}$

The final attendance rate $(42.5 \%$ overall wit $\overrightarrow{\mid T}$ protocol 1 and $46 \%$ with protocol 2) was higher than the spontaneous response rates, showing the impact of a follow-up telephone call after a written invitation. As with the spontaneous response rate approaching the adolescent first (protocol 2) di not improve the attendance rate.

Of note is the very high percentage of teenagere who agreed to attend the visits after being called (79\% overall with protocol $1,73 \%$ overall wit protocol 2). Many of these patients did not atten but had agreed to come, possibly because of diffio culty refusing their family nurse or physician. O the other hand, many of those agreeing but no attending might be part of a large population of teenagers who would be interested in preventive health visits but are not highly motivated to attend For them, using an opportunistic approach (ap) proaching them when they attend the clinic fog other reasons) might be a reasonable invitatiois strategy.

The attendance rates that we have reported are similar to those reported previously in a genera practice setting in the United Kingdom. ${ }^{8}$ Nonethe less, they are lower than we had anticipated, espe 0 cially in the context of a family practice clinic witkw generally close and long-term relationships be tween providers and patients. This lower rate्छ might reflect the actual interest level in preventive health care visits, concerns regarding confidential ${ }^{\circ}$ ity, an unfamiliarity with preventive adolescent vis its on the part of both teenagers and their parents in this population, or the proclivity of teenagers not to show up for their scheduled appointments. 
This pilot study shows the potential for bringing nearly one half of an adolescent patient population unaccustomed to preventive health care to a family practice clinic for comprehensive visits. It is important to determine which adolescents attend preventive visits - by demographic categories (including socioeconomic status and ethnic group) and by risk status. These analyses, for a larger sample of the same population, will be reported in a future article and might reveal additional demographic differences that were not statistically significant for this initial sample. Determining the extent to which we reach the most needy adolescents and developing strategies to improve compliance for these groups ought to be important objectives for every primary care provider working with adolescents.

The authors gratefully acknowledge the participation in the program of family nurses Galit Cnaan, Hana Gordon, Miri Haringman, Pnina Naveh, and Aliza Velber.

\section{References}

1. Elster AB, Kuznets NJ, editors. AMA guidelines for adolescent preventive services (GAPS): recommen- dations and rationale. Baltimore: Williams \& Wilkins, 1994.

2. Knishkowy B, Palti H. GAPS (AMA guidelines for adolescent preventive services). Where are the gaps? Arch Pediatr Adolesc Med 1997;151:123-8.

3. Knishkowy B, Schein M, Palti H, Cnaan G, Naveh P. Community-oriented adolescent care in an Israeli family practice: background and beginnings. Presented at the Second European Forum on Adolescent Health; June 23, 1993; Jerusalem, Israel.

4. Neinstein LS. adolescent health care: a practical guide. 3rd ed. Baltimore: William \& Wilkins, 1996: 51-2.

5. Mehta C, Patel N. StatXact-4 for windows. Cambridge, Mass: Cytel Software, 1999.

6. Balancing alliances. Family-oriented care of adolescents. In: McDaniel SH, Campbell TL, Seaburn DB. Family-oriented primary care: a manual for medical providers. New York: Springer-Verlag, 1990:15166.

7. Cheng TL, Savageau JA, Sattler AL, DeWitt TG. Confidentiality in health care. A survey of knowledge, perceptions, and attitudes among high school students. JAMA 1993;269:1404-7.

8. Campbell A, Edgar S. Teenage screening in a general practice setting. Health Visit 1993;66:365-6.

\section{Appendix 1}

\section{Letter of Explanation to Parents}

Dear Parents,

The doctors and nurses in the Family Practice Unit of the Hadassah Community Health Center have conducted health programs on various topics for many years. Our experience with teenagers aged 12 to 18 years has shown that this is a healthy population that visits the health center infrequently. On the other hand, they have health and behavioral needs that could be helped by meeting with the medical staff for prevention and health education. Therefore, we are inviting every adolescent in the $7^{\text {th }}$ and $10^{\text {th }}$ grade for an individual visit with the family nurse and physician, in which we relate to issues of importance to them and also to a number of other health issues. The visit includes a questionnaire, discussions with the nurse and physician, and a physical examination.

We request your cooperation in this health promotion program for your child. Please fill out the following pages to let us know which health topics, in your opinion, are important to bring up in the meeting with your child.

We are concurrently sending an invitation to your child, to visit us in the health center, and request that you sign the Consent Form that $s /$ he will receive for participation in the program.

(Note: Following the above letter is a checklist of 44 topics that the parents can request that we discuss during the health visit, 3 of which they can rate as being most important. There is also a question asking parents whether they would like a separate meeting with the medical team.)

Sincerely yours,

Dr.

Family Nurse 


\section{Appendix 2}

Invitation Letter to Teenager and Consent Form

Dear

The Family Practice staff of the Hadassah Community Health Center are pleased to invite you for a visit to the health center to get to know you better. The meeting will include a short, selfadministered questionnaire, a brief physical examination, and a discussion with the family nurse and doctor about various health topics.

The meeting will take place during the afternoon hours in the health center, and will last around 40 minutes.

Please call the clinic (phone number -) in the next few days to set up a time for the visit. Also, please bring the attached parental consent form with you to the visit.

Sincerely,

Dr.

Family Nurse

\section{Consent Form}

1. I agree to my son/daughter's participation in the adolescent health program in the family practice center.

2. Dr. and family nurse have explained the program's content to me, and that this includes filling out a questionnaire, a discussion with the nurse and doctor, and a physical examination, in the course of which attention will be given to a number of adolescent health topics.

Father's signature Date

Mother's signature Date 